\title{
Reconstruction of Twist Torque in Main Parachute Risers
}

\author{
Joshua D. Day ${ }^{1}$ \\ Embry-Riddle Aeronautical University, Prescott, AZ, 86301
}

The reconstruction of twist torque in the Main Parachute Risers of the Capsule Parachute Assembly System (CPAS) has been successfully used to validate CPAS Model Memo conservative twist torque equations. Reconstruction of basic, one degree of freedom drop tests was used to create a functional process for the evaluation of more complex, rigid body simulation. The roll, pitch, and yaw of the body, the fly-out angles of the parachutes, and the relative location of the parachutes to the body are inputs to the torque simulation. The data collected by the Inertial Measurement Unit (IMU) was used to calculate the true torque. The simulation then used photogrammetric and IMU data as inputs into the Model Memo equations. The results were then compared to the true torque results to validate the Model Memo equations. The Model Memo parameters were based off of steel risers and the parameters will need to be re-evaluated for different materials. Photogrammetric data was found to be more accurate than the inertial data in accounting for the relative rotation between payload and cluster. The Model Memo equations were generally a good match and when not matching were generally conservative.

\section{Nomenclature}

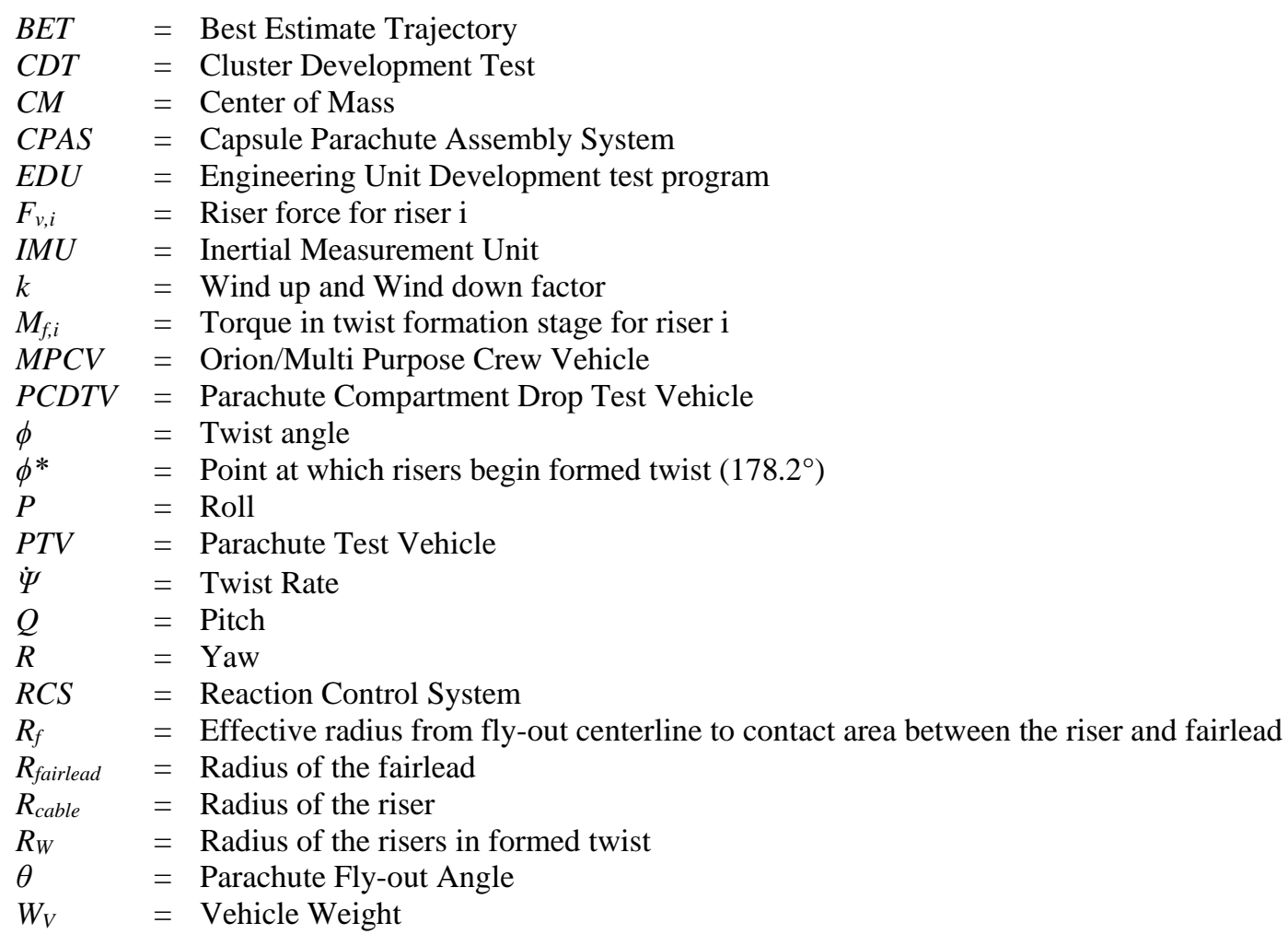

\footnotetext{
${ }^{1}$ Student, Embry-Riddle Aeronautical University, 3700 Willow Creek Rd., Prescott, AZ 86301, AIAA Member. 


\section{Introduction}

A 11 of the parachute types that Orion is to use are deployed in the current Engineering Unit Development (EDU) test program. The Main parachutes that are deployed last in the sequence are used to slow the spacecraft to its final descent rate and aid in achieving a proper orientation prior to splashdown. In order to achieve a proper orientation the twist torque acting on the system must be known. A proper orientation is required for the safety of the Orion crew. If an undesired orientation occurs it could cause damage to the capsule and possibly loss of crew members. The structural capabilities and hydro-dynamic loading as the capsule decelerates against the water requires that the Orion 'edge into the water'. Therefore, it is important to achieve the correct orientation on splashdown. The validated Model Memo equations will be used to run Monte Carlo sets to predict possible twist torques that will be applied to the capsule. The torque data will then be used to predict the amount of fuel required for the Reaction Control System (RCS) thrusters to counteract the twist torque and provide a favorable orientation. This will ensure that the Orion capsule has achieved the proper orientation upon splashdown.

Phase I of developing a twist torque simulation evaluated a Generation II Weight Tub test vehicle. This vehicle and Cluster Development Test (CDT) 2-2 was used for simplicity. CDT-2-2 had visible twist and involved only 1DOF. Phase I validated the torque reconstruction technique and provided the rational to continue to Phase II. Phase II began the evaluation of EDU tests. The Phase II test vehicle was a Parachute Compartment Drop Test Vehicle (PCDTV) (for CPAS, a dart shaped vehicle). The PCDTV created the most twist, which helped to further validate the parameters of the Model Memo Torque Equation. Phase III evaluated the data from a Parachute Test Vehicle (PTV), which is the closest in similarity to the Orion/Multi Purpose Crew Vehicle (MPCV). The Model Memo Torque equation and the simulation were further validated.

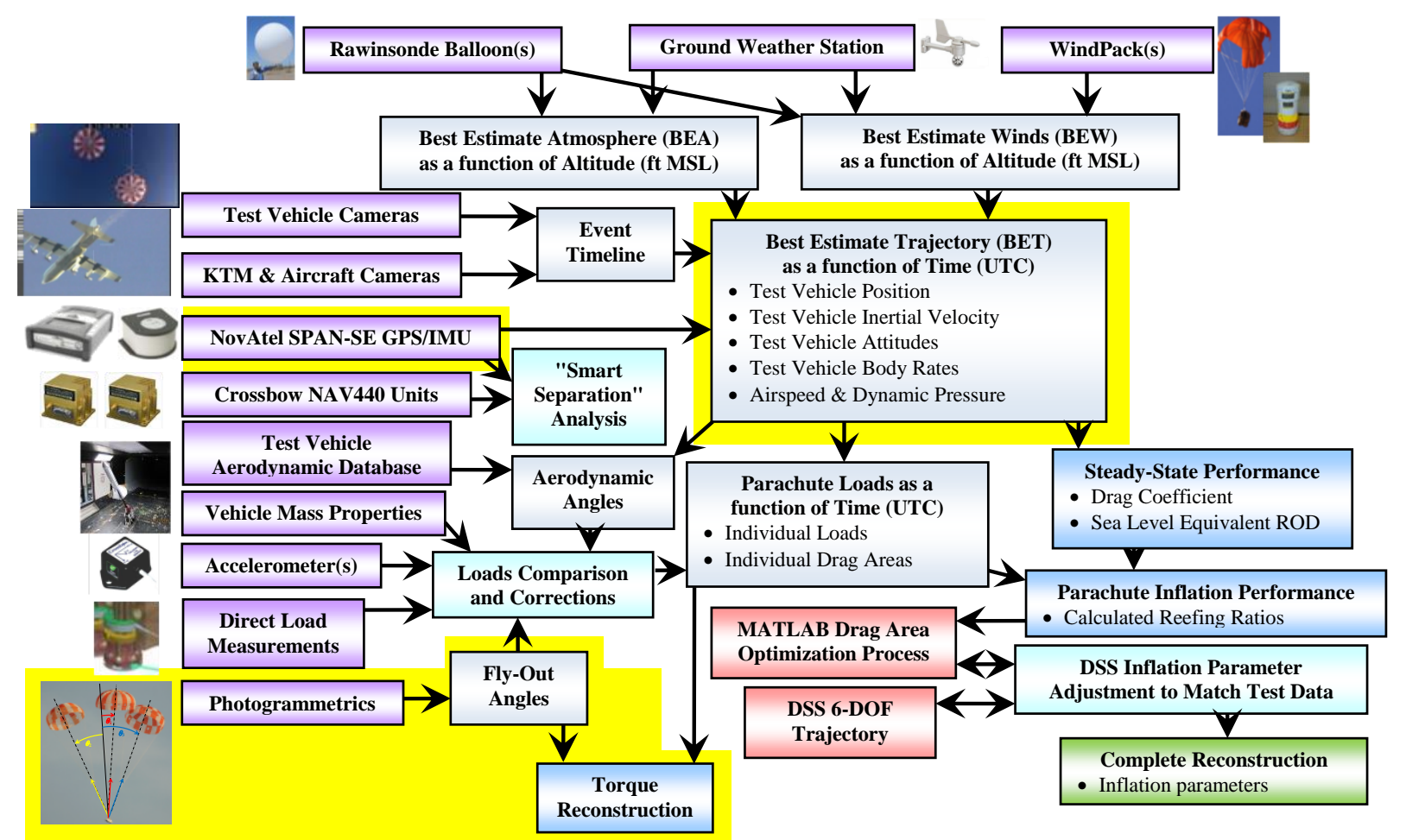

Figure 1. Flight test reconstruction process with torque reconstruction features highlighted in yellow.

As shown in Fig. 1, the true torque was calculated using IMU data. The IMU data was used to create a Best Estimate Trajectory (BET) that includes the test vehicle inertia, attitudes and body rates. The twist acceleration about the vertical axis was calculated and then multiplied by the inertia in order to get the true twist torque. A combination of the IMU data and photogrammetrics was then used. The integral of the twist rate from the IMU data provided the twist angle and the photogrammetrics provided the fly-out angles. The twist angle and the fly-out angles were then used in the Model Memo Torque Equation to calculate the twist torque. Then, only photogrammetric data was used to 
compute the fly-out angles and the twist angle. The Model Memo Torque Equation was then used again to calculate the twist torque. The values from the true torque were then compared to the two values given by the Model Memo equation to ensure a good match and validate the Model Memo Conservative Torque Equation.

\section{Equations and Assumptions}

There are two phases of twisting. The first phase is called the "twist formation" phase ${ }^{[1]}$. The "twist formation" phase is characterized under low twist angles, where a torque is applied by the friction of the risers on the fairlead. As a riser is pulled against the edge of the fairlead, it causes a moment to be formed on the CM and a twist to begin forming about the fly-out axis $(\theta)$.

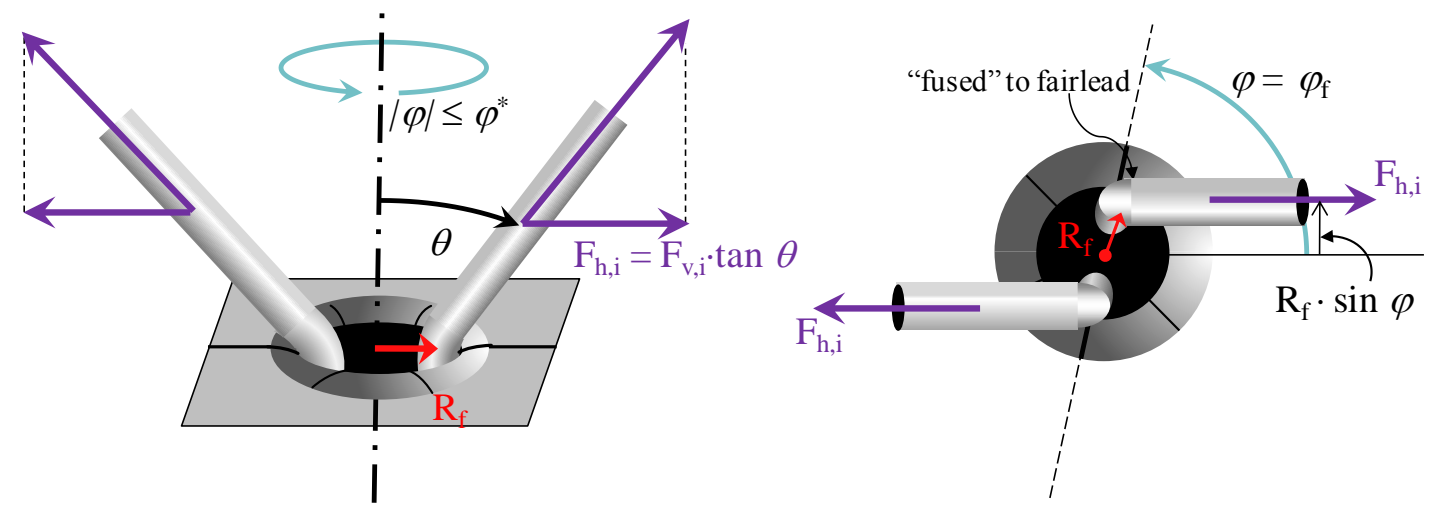

Figure 2. Twist formation (2-risers).

The torque during the twist formation stage is given by Eq. 1:

$$
M_{f, i}=-R_{f} \cdot F_{v, i} \cdot \tan (\theta) \cdot \sin \left(\phi_{f}\right)
$$

$R_{f}$ is the effective radius from the centerline to the contact area between the riser and the fairlead. $R_{f}$ is given by Eq. 2:

$$
\mathrm{R}_{\mathrm{f}}=\mathrm{R}_{\text {fairlead }}-\mathrm{R}_{\text {cable }}
$$

$\mathrm{F}_{\mathrm{v}, \mathrm{i}} \cdot \tan (\theta)$ is the horizontal component of the riser force acting on the fairlead. In steady state conditions the conservative summation of riser tension vertical components equals the weight of the vehicle therefore the expression becomes Eq. 3:

$$
\mathrm{M}_{\mathrm{f}} \approx-\mathrm{W}_{\mathrm{V}} \cdot \tan \theta \cdot \mathrm{R}_{\mathrm{f}} \cdot \sin \phi
$$

The factor $\sin \left(\phi_{\mathrm{f}}\right)$ accounts for the effect of the twisting rotation of the risers about the fly-out axis. Due to the fused and slipped conditions empirically found in the twist formation stage the torque curve must be stretched by multiplying the twist angle by $3 / 4$ to calculate an estimated effective fairlead twist angle. This implies that $\phi_{\mathrm{f}} \approx \frac{3}{4} \phi$. Therefore, the twist formation expression becomes Eq. 4:

$$
\mathrm{M}_{\mathrm{f}} \approx-\mathrm{R}_{\mathrm{f}} \cdot \mathrm{W}_{\mathrm{V}} \cdot \tan (\theta) \cdot \sin \left(\frac{3}{4} \phi\right)
$$

American Institute of Aeronautics and Astronautics 
The second phase is the "formed twist" phase. The formed twist phase begins after the risers rotate enough to start wrapping around each other.
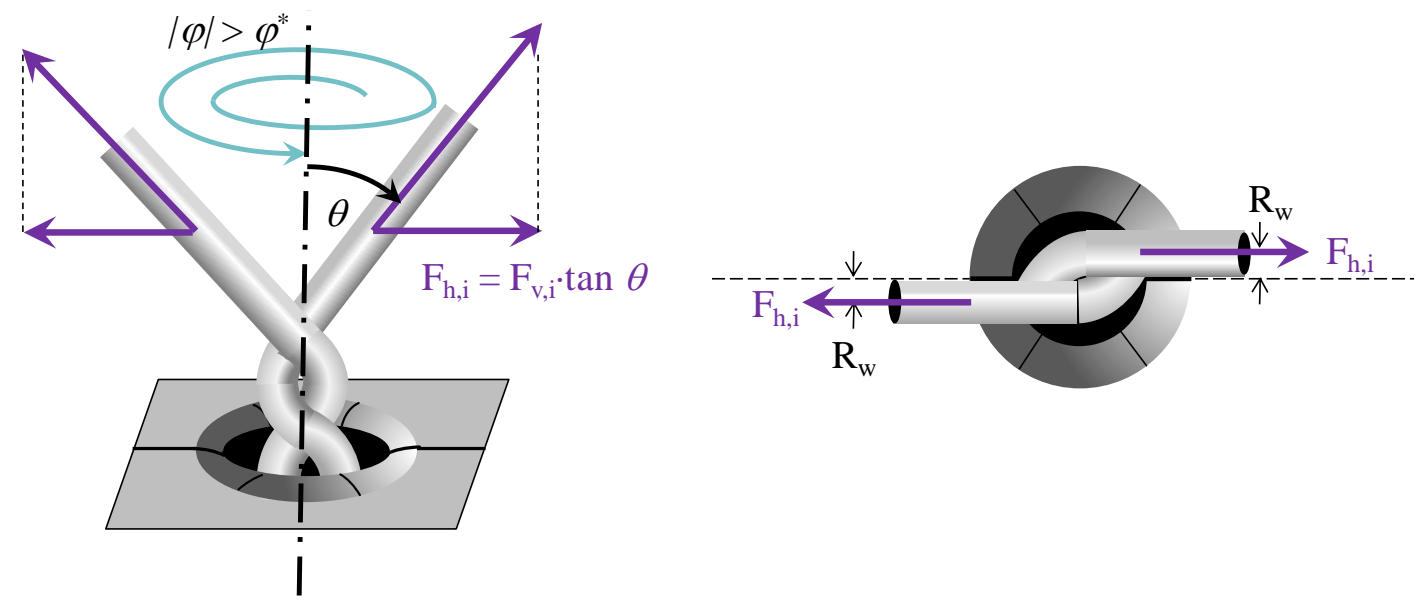

Figure 3. Formed twist diagram.

A new torque radius is developed based on the geometry of the twisted risers. The base formed twist torque is given by the expression:

$$
\mathrm{M}_{\mathrm{f}} \approx-\mathrm{R}_{\mathrm{W}} \cdot \mathrm{W}_{\mathrm{V}} \cdot \tan (\theta)
$$

The new radius that is formed, $\mathrm{R}_{\mathrm{W}}$, is dependent on the riser thickness and the riser geometry in the twisted phase. Based on a relationship between riser diameter and a formed twist phase theoretical radius, $\mathrm{R}_{\mathrm{W}}$, changes with the number of risers. This is shown below, where $\mathrm{R}$, is the measured riser radius.

$$
\begin{array}{cc}
\text { 2-Riser } & \mathrm{R}_{\mathrm{W}, \text { theoretical }}=\mathrm{R} \\
\text { 3- } \text { Riser } & \mathrm{R}_{\mathrm{W} \text {,theoretical }}=1.15 \mathrm{R}
\end{array}
$$

There is a difference in torque while being wound up as opposed to being unwound. Experimental data showed that the relationship was $\mathrm{M}_{\mathrm{up}} \cong \mathrm{k} \cdot \mathrm{M}_{\mathrm{down}}$, where $\mathrm{k}=1.3$. An experimental method to determine the effective radius of the twisted risers based on testing geometry was developed. This experimental radius was determined using Eq. 6 below. The experimentally determined radius, $\mathrm{R}_{\mathrm{W} \text {, exp }}$, had a smaller value than the theoretically calculated values, but matched ground test data better. Differences between theoretical and experimental radius values are due to the interaction between the risers. As the risers wind around each other, the steel ropes tend to flatten out.

$$
\mathrm{R}_{\mathrm{W}, \exp }=\frac{\mathrm{dh}}{\mathrm{d} \phi} \frac{1}{\tan \left(\theta_{0}\right)}
$$

Figure 4 shows the differences in how well the experimental and theoretical radii match the test data. The experimentally determined radius (dashed black line) provides a closer match for wind-up data, while the theoretical radius provides a conservative value. 


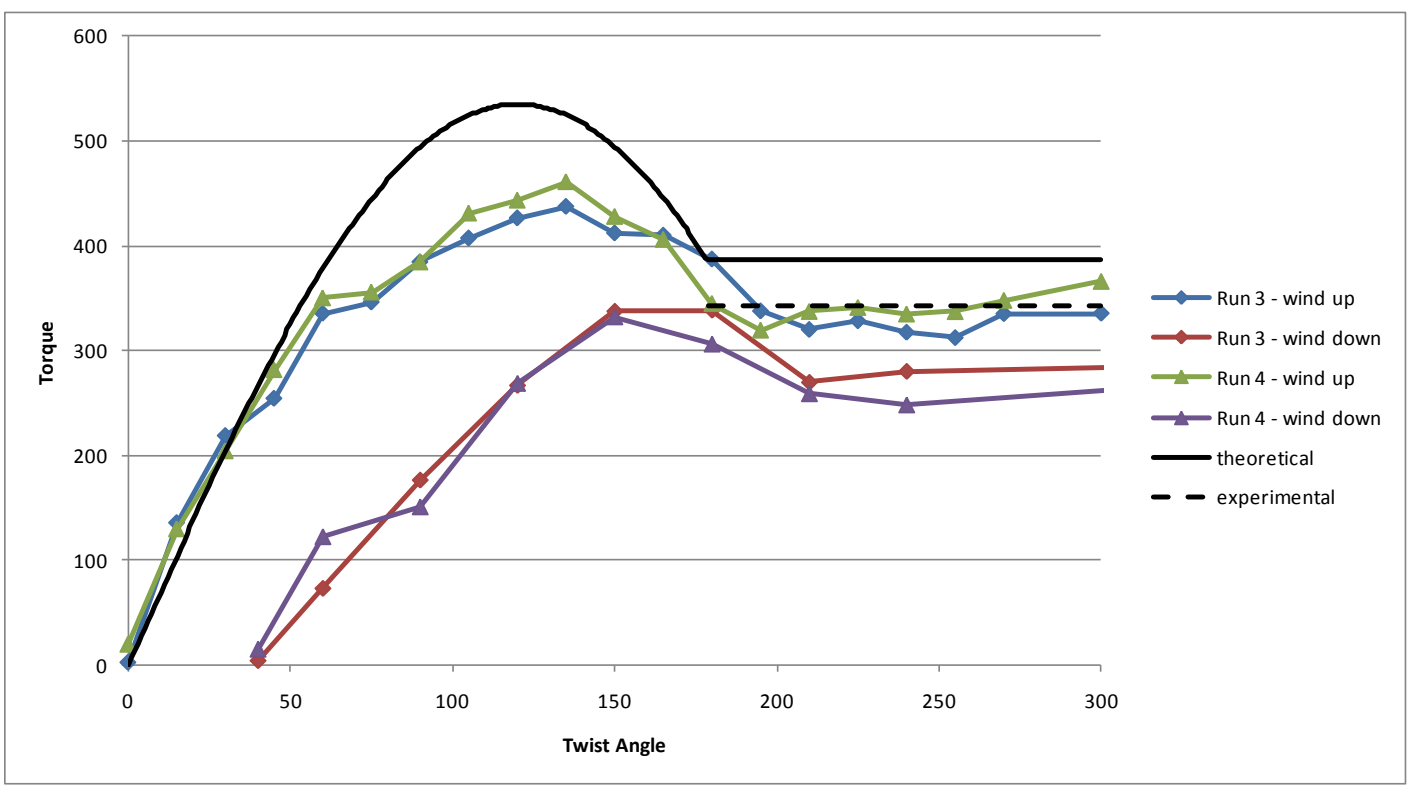

Figure 4. Consertiave model and $\theta=15.5^{\circ}$ ground test data

The conservative torque equation becomes Eq. 7:

$$
\mathrm{M}_{\mathrm{f}}= \begin{cases}-\mathrm{R}_{\mathrm{f}} \cdot \mathrm{F}_{\mathrm{v}} \cdot \tan (\theta) \sin \left(\frac{3}{4} \phi\right), & \text { if }|\phi|<\phi^{*} \\ -\mathrm{k} \cdot \mathrm{R}_{\mathrm{W}} \cdot \mathrm{F}_{\mathrm{v}} \cdot \tan (\theta) \cdot \operatorname{sign}(\phi), & \text { if }|\phi| \geq \phi^{*}\end{cases}
$$

Where:

$\mathrm{F}_{\mathrm{v}} \approx$ Suspended Vehicle Weight $\left(\mathrm{W}_{\mathrm{v}}\right)$

$\mathrm{R}_{\mathrm{W}}=$ effective radius of the twisted risers

$\mathrm{R}_{\mathrm{f}}=$ effective radius from the centerline to the contact area between the riser and the fairlead

$\mathrm{k}=1.3$ for $\dot{\phi} \geq 0,1.0$ for $\dot{\phi}<0$

$\phi^{*}=178.2^{\circ}$

The twist formation phase is represented by the first part of Eq. 7 and the formed twist phase begins at $\phi^{*}$ and is defined by the second part of Eq. 7. The $\phi$ angle was determined by both the IMU data and photogrammetrics. Figure 5 and Eq. 8 show how photogrammetrics were used to calculate $\phi$.
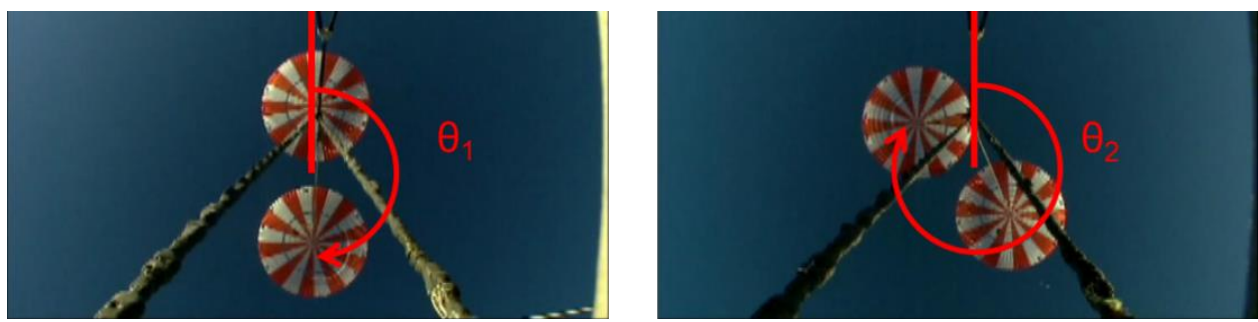

Figure 5. Photogrammetric $\phi$ angle.

$$
\theta_{2}-\theta_{1}=\phi
$$

Figure 6 shows the body rates $\mathrm{P}, \mathrm{Q}$, and R and the assumed twist rate $(\dot{\Psi})$ axis.

American Institute of Aeronautics and Astronautics 


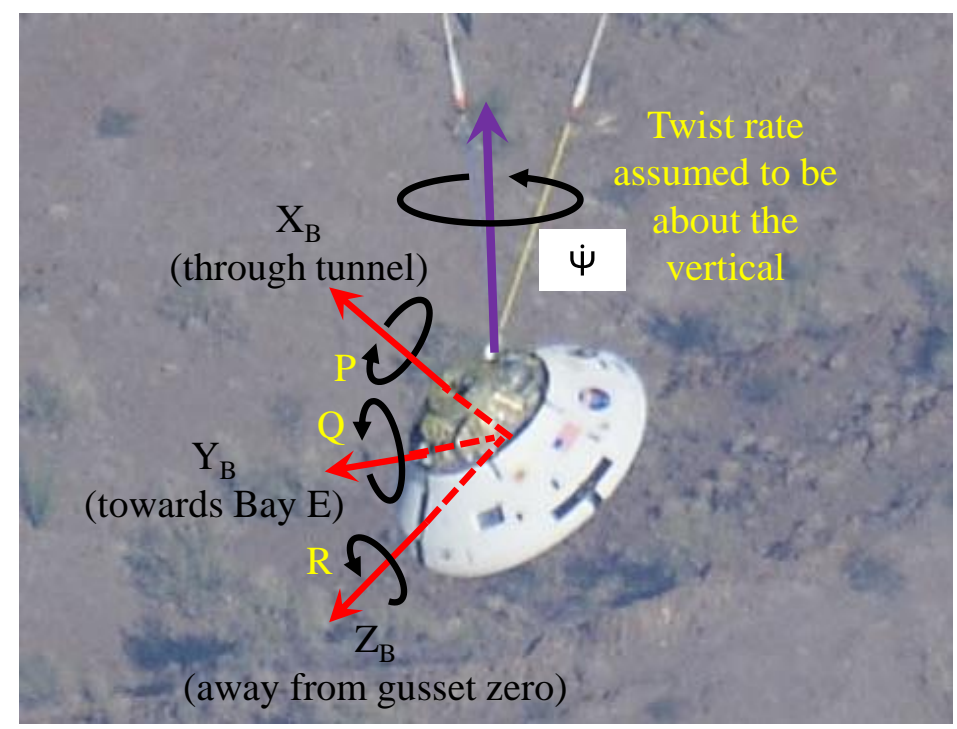

Figure 6. Twist rate compared to body rates.

The twist rate is calculated using the pitch, roll, yaw, and their rates from the IMU data as shown in the following rates transformation Eq. 9:

$$
\begin{gathered}
{\left[\begin{array}{c}
\dot{\phi} \\
\dot{\theta} \\
\dot{\Psi}
\end{array}\right]=\left[\begin{array}{ccc}
1 & 0 & 0 \\
0 & \cos (\text { roll }) & -\sin (\text { roll }) \\
0 & \sin (\text { roll }) & \cos (\text { roll })
\end{array}\right] *\left[\begin{array}{ccc}
\cos (\text { pitch }) & 0 & \sin (\text { pitch }) \\
0 & 1 & 0 \\
-\sin (\text { pitch }) & 0 & \cos (\text { pitch })
\end{array}\right] *} \\
{\left[\begin{array}{ccc}
\cos (\text { yaw }) & -\sin (\text { yaw }) & 0 \\
\sin (\text { yaw }) & \cos (\text { yaw }) & 0 \\
0 & 0 & 1
\end{array}\right] *\left[\begin{array}{c}
P \\
Q \\
R
\end{array}\right]}
\end{gathered}
$$

The derivative of the twist rate is then multiplied by the inertia to calculate the true torque, such that $\mathrm{M}_{\mathrm{f}}=[\ddot{\Psi}] *[I]$. The twist moments are given by the Eq. 10:

$$
\left[\begin{array}{l}
M_{x} \\
M_{y} \\
M_{z}
\end{array}\right]=[I] *\left[\begin{array}{c}
\dot{P} \\
\dot{Q} \\
\dot{R}
\end{array}\right]+\left(\left[\begin{array}{l}
P \\
Q \\
R
\end{array}\right] \times[I] *\left[\begin{array}{l}
P \\
Q \\
R
\end{array}\right]\right)
$$

\section{Test Reconstruction}

\section{A. Phase I: CDT-2-2 Platform Test}

The first test to be reconstructed was used for simplicity and to validate the reconstruction technique. The test was a platform test with 1-DOF, no confluence, and it was stable. The first step to reconstruct the twist torque was to look at video footage of the test drops and determine the times at which each phase began and ended and when there was wind up or wind down. The lack of confluence caused the $\mathrm{R}_{\mathrm{f}}$ parameter to be zero and only the second function in the Model Memo Conservative Torque Equation: Eq. 7, to be used. The data was then run through the simulation according to Fig. 7. 


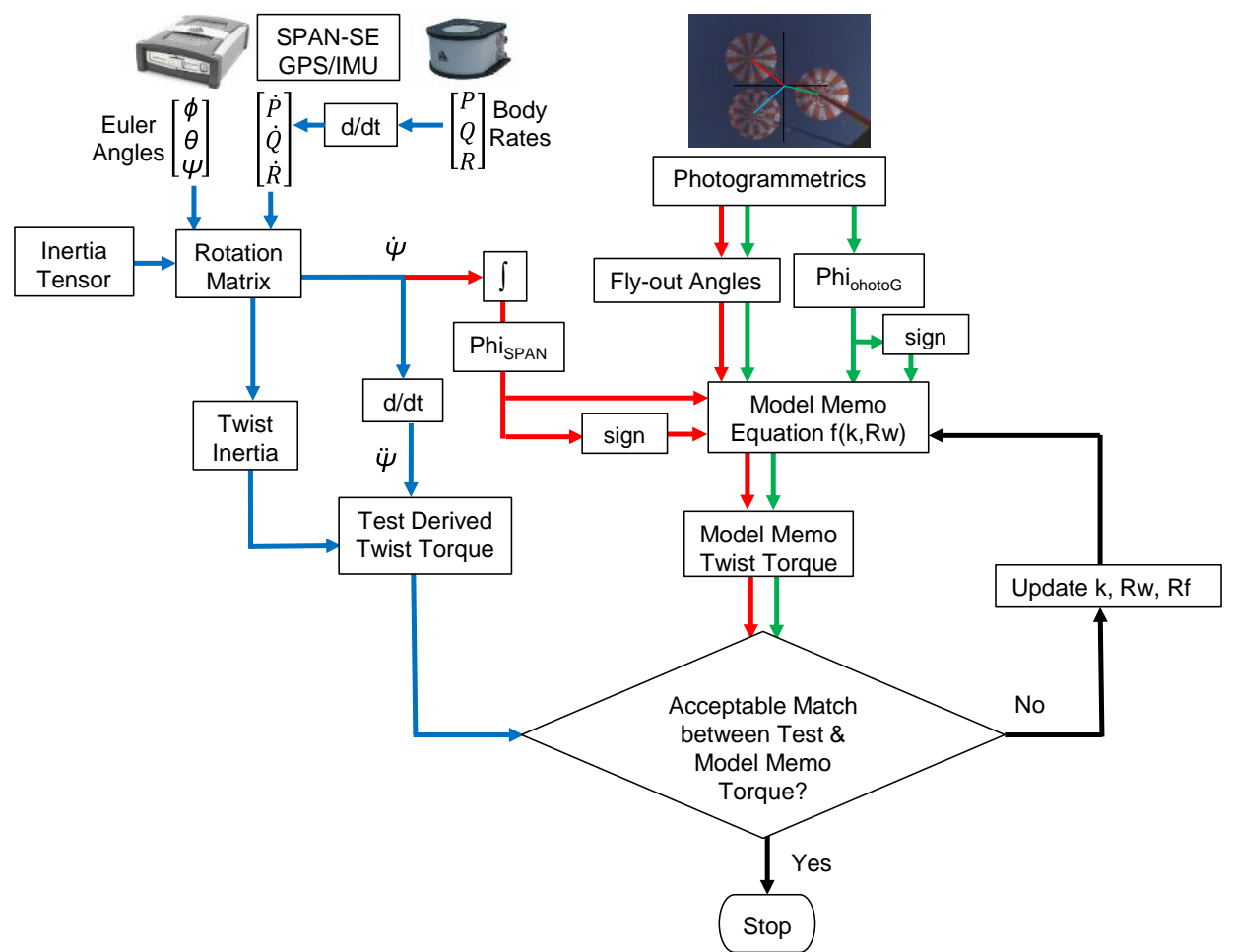

Figure 7. Simulation Methodology.

The twist angles $(\phi)$ from the SPAN-SE and the photogrammetrics were compared to ensure a good match. They were also adjusted to be at zero twist at the same times that the video showed zero twist. Once a good match was made the simulation was run again to calculate the twist torque.
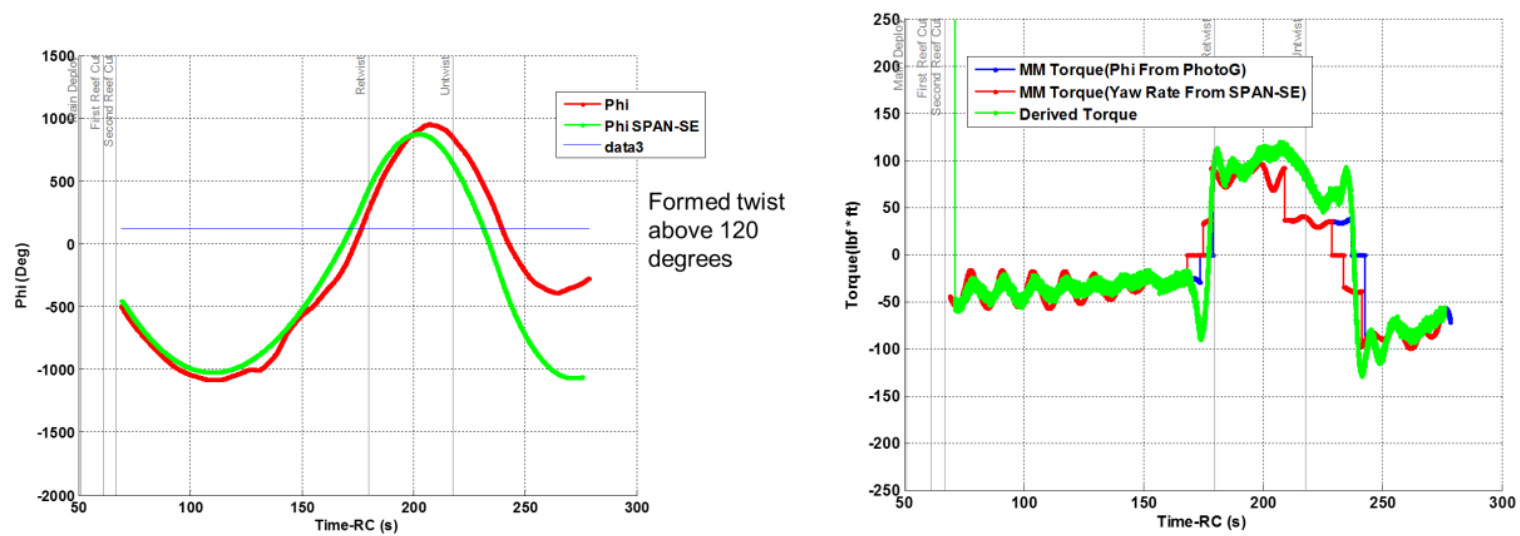

Figure 8. Twist angle and Twist Torque Reconstructions.

As Fig. 8 shows, there was a good match between the true torque or "derived torque" (in green) and the Model Memo torque equations (blue and red). It can also be seen that the Model Memo equations are indeed conservative. CDT-2-2 had only two main parachutes on a confluence, which caused the formed twist to start when the twist angle was above $120^{\circ}$ instead of $178.2^{\circ}$ in a fairlead. This data proved that the technique of reconstruction was viable and the Model Memo Torque Equation was accurate enough to match the true torque. This provided the rational to continue to Phase II and begin reconstruction of EDU tests.

\section{B. Phase II: CDT-3-1 and CDT-3-2 PCDTV Test}

American Institute of Aeronautics and Astronautics 
1. CDT-3-1

The EDU tests including Phase II and Phase III test had a single attach point for all of the risers, through a fairlead, resulting in a variable hang angle. Due to the hang angle the EDU tests had coupled roll, pitch and yaw. The EDU tests also had a fairlead so the full Model Memo Torque Equation was used. Phase II used the PCDTV as shown in Fig. 9.

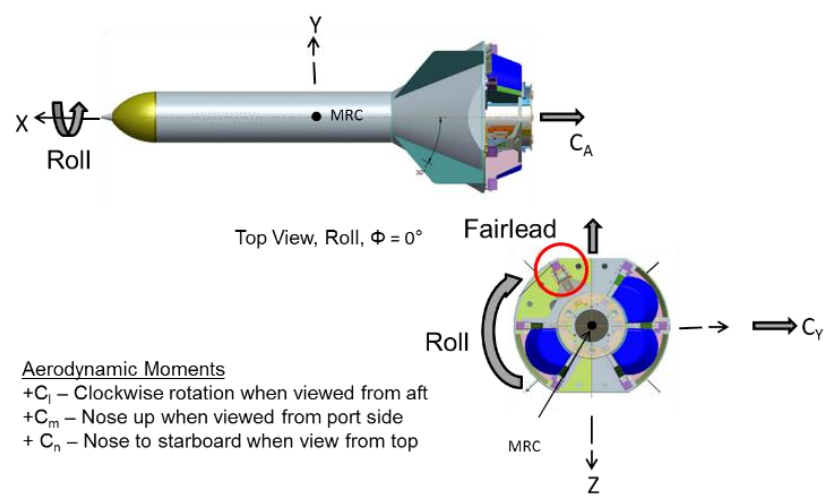

Figure 9. PCDTV Coordinate System.

The video footage of the drop tests was reviewed and timelines were made showing when the risers were twisting up, twisting down, and untwisted. The data was run through the simulation using basically the same methodology as Phase I. The only difference in the methodology was instead of just yaw rate being used to calculate the twist torque pitch and roll were also included using Eq. 9 to calculate the twist rate (广). The twist angle was adjusted to be equal to zero at the points where the risers were completely untwisted. Figure 10 shows the new methodology and that a good match was achieved for the twist angle.
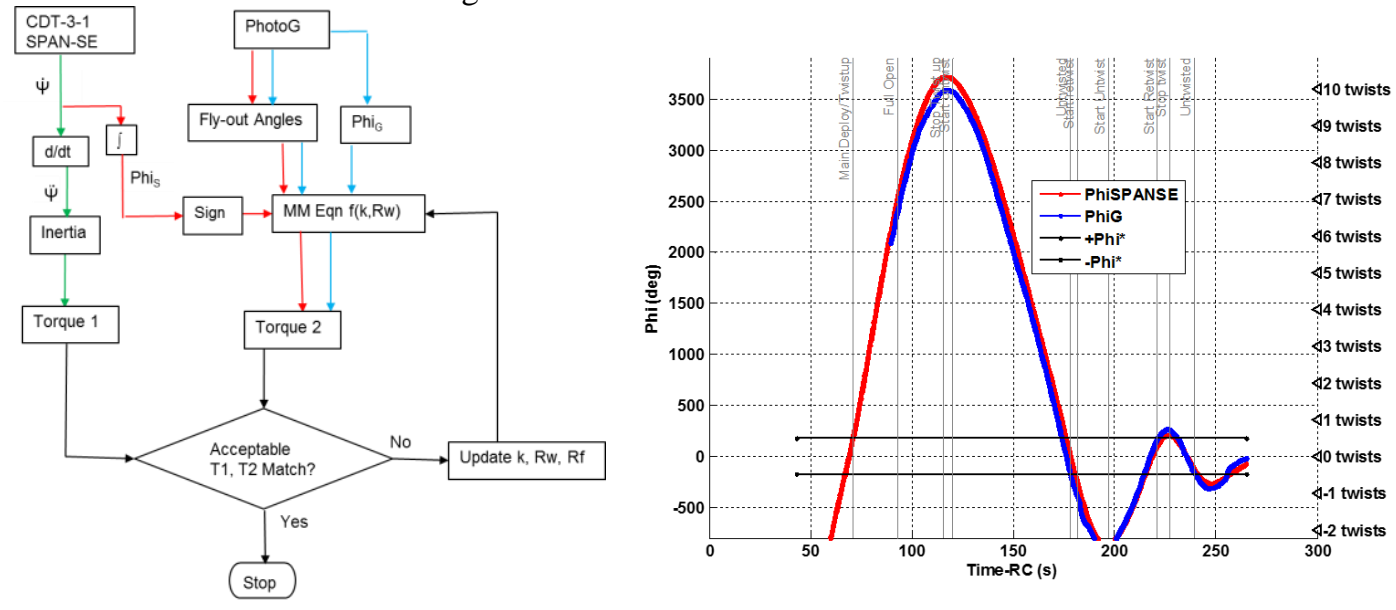

Figure 10. Methodology and Twist Angle.

The Phi SPANSE (red trace in Fig. 10) was calculated by integrating the twist rate whereas the PhiG (blue trace in Fig. 10) was calculated using photogrammetrics. The horizontal bars of \pm Phi* are at $\pm 178.2^{\circ}$. Phi* is the point where formed twist begins and between \pm Phi* there is no formed twist.

Most of the total body rate came from the roll rate but due to the hang angle the pitch and yaw rates also affected the total body rate as shown in Fig. 11. In Fig. 11 the mains fully open at about 95 Time-RC (s), any body rates before that time were not taken into account because the parachutes were not yet open. The twist rate was derived using the attitude data and Eq. 9. The twist rate was then used to find the torque about the twist axis.

American Institute of Aeronautics and Astronautics 

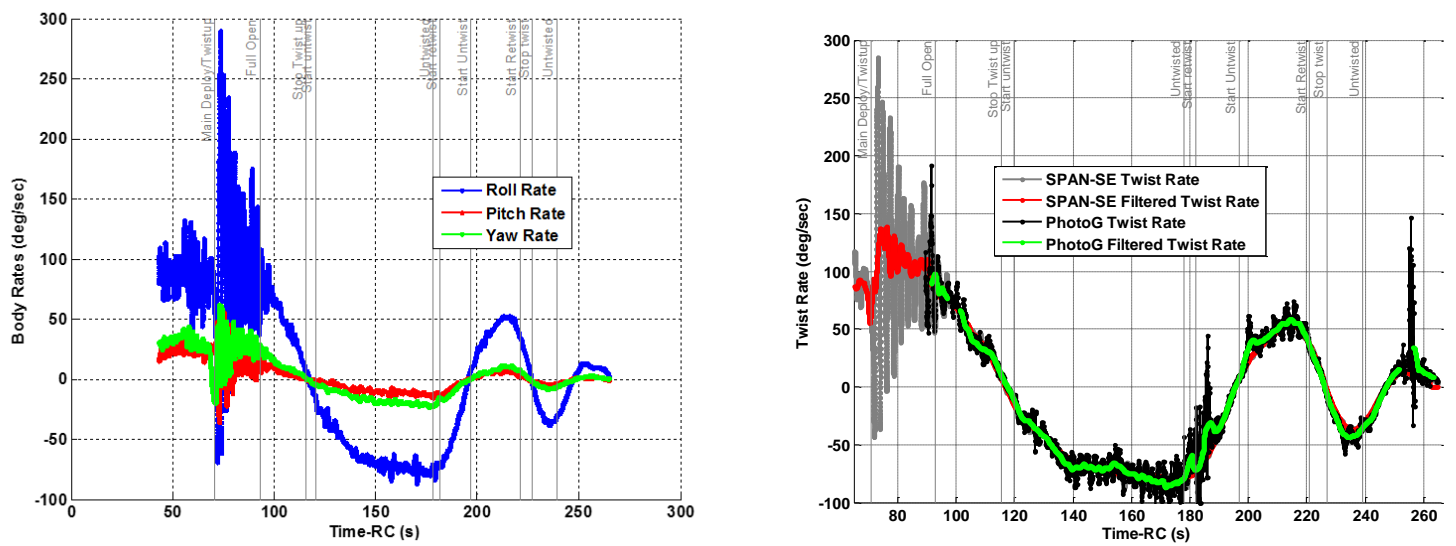

Figure 11. CDT-3-1 Body Rates and Roll/Twist Rates.

The simulation was then run to calculate the true or reconstructed twist torque (blue trace in Fig. 12) and the Model Memo twist torque (green and red traces in Fig. 12). They were a good match, and the Model Memo predicted torque proved to be conservative but follow the true torque trend very well.

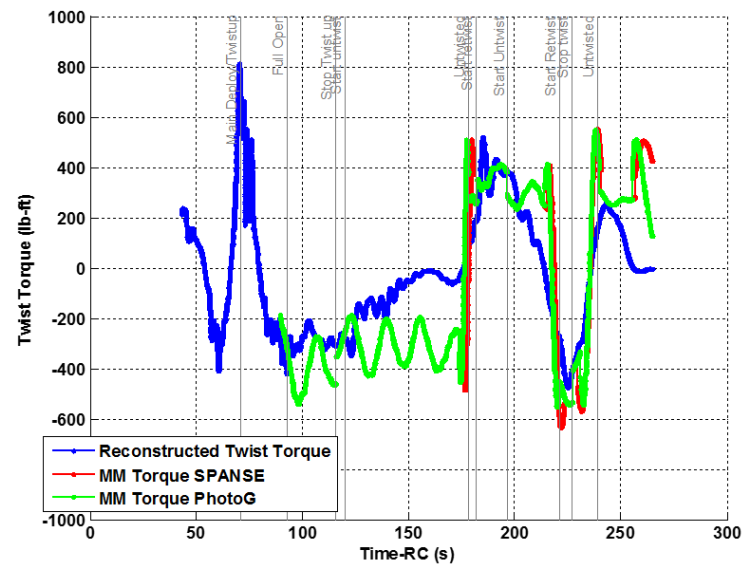

Figure 12. CDT-3-1 Twist Torque.

2. CDT-3-2

CDT-3-2 used the same method as CDT-3-1. The twist rate was again almost all created by the roll as shown by the flight test data in Fig. 13.

American Institute of Aeronautics and Astronautics 

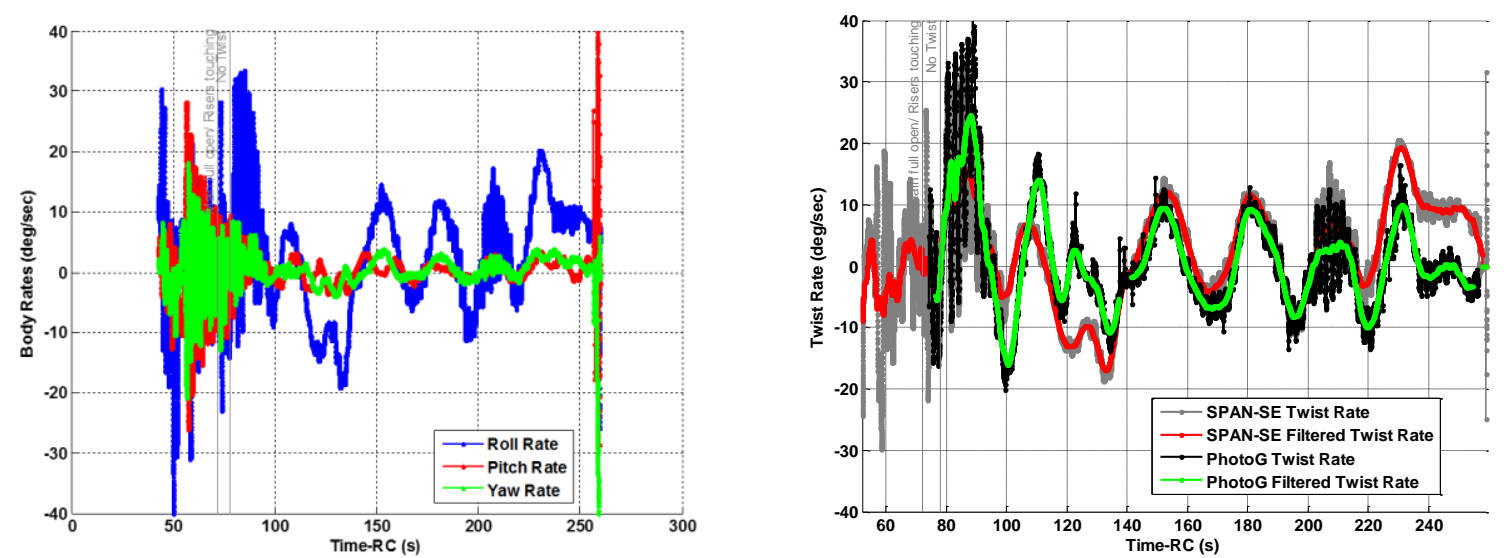

Figure 13. CDT-3-2Body Rates and Roll/Twist Rate.

The IMU data showed a complete twist in the last minute of flight but after review of the video footage it was discovered that the parachutes and the PCDTV rotated at the same speed during that time and there was no twist. The IMU reads what the PCDTV does and does not account for the movement of the parachutes, whereas the photogrammetrics uses the relative motion of the chutes to the body. The discrepancies between the twist angle using the IMU or SPAN-SE data and the twist angle using photogrammetrics is shown in Fig. 14. The simulation was then run to calculate the twist torque. The Model Memo torque using the photogrammetric data provided a good match to the true torque and was once again conservative.
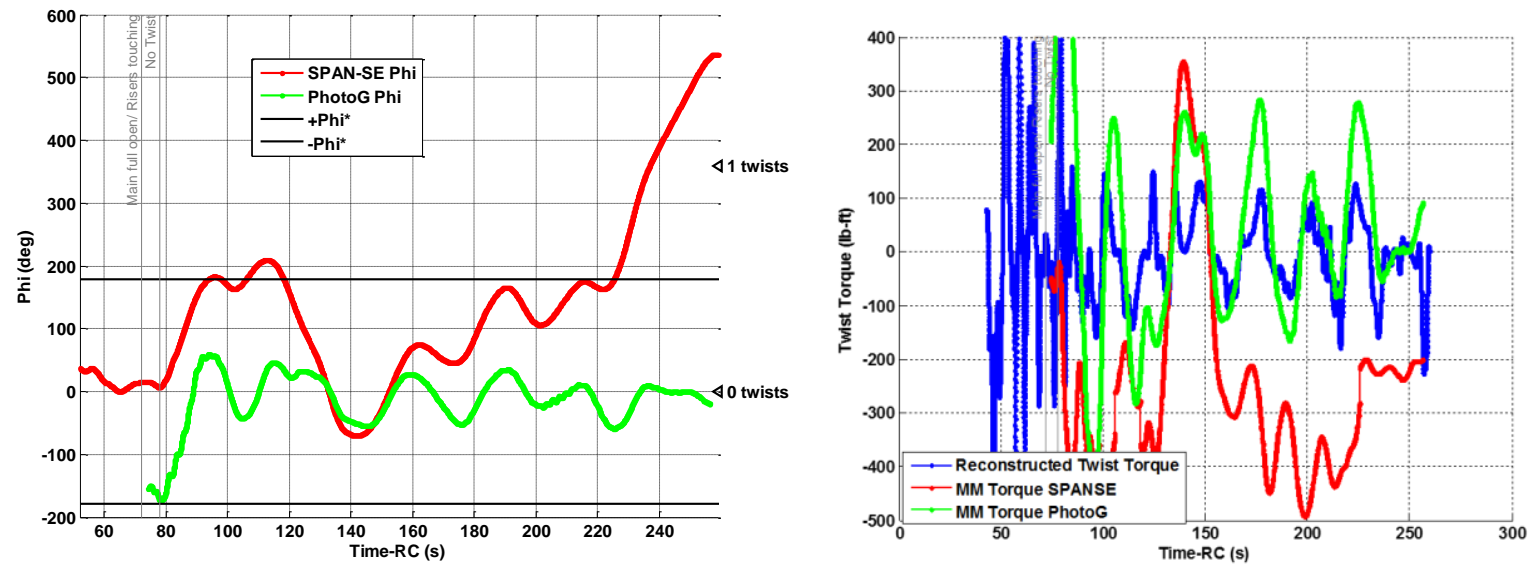

Figure 14. CDT-3-2 Twist Angle and Twist Torque.

\section{Phase III: CDT-3-3 and CDT-3-5 PTV Test}

The PTV is a truncated capsule that is the closest in similarity to the Orion/Multi Purpose Crew Vehicle. The axis system is also slightly changed between the PCDTV and PTV as shown in Fig. 15. The exact same methodology was used between Phase II and Phase III the only difference being the test vehicles. Due to the change in the axis system the yaw rate had a significant increase in effect on the twist rate as shown in Fig. 16. In Fig. 16 at about T-RC 95 the main steady state phase of flight began. The twist rate and twist angle were also calculated in the same way as in Phase II.

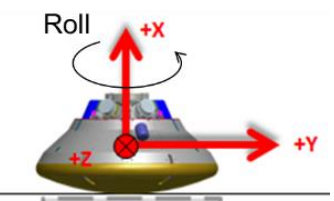

Figure 15. PTV System. 

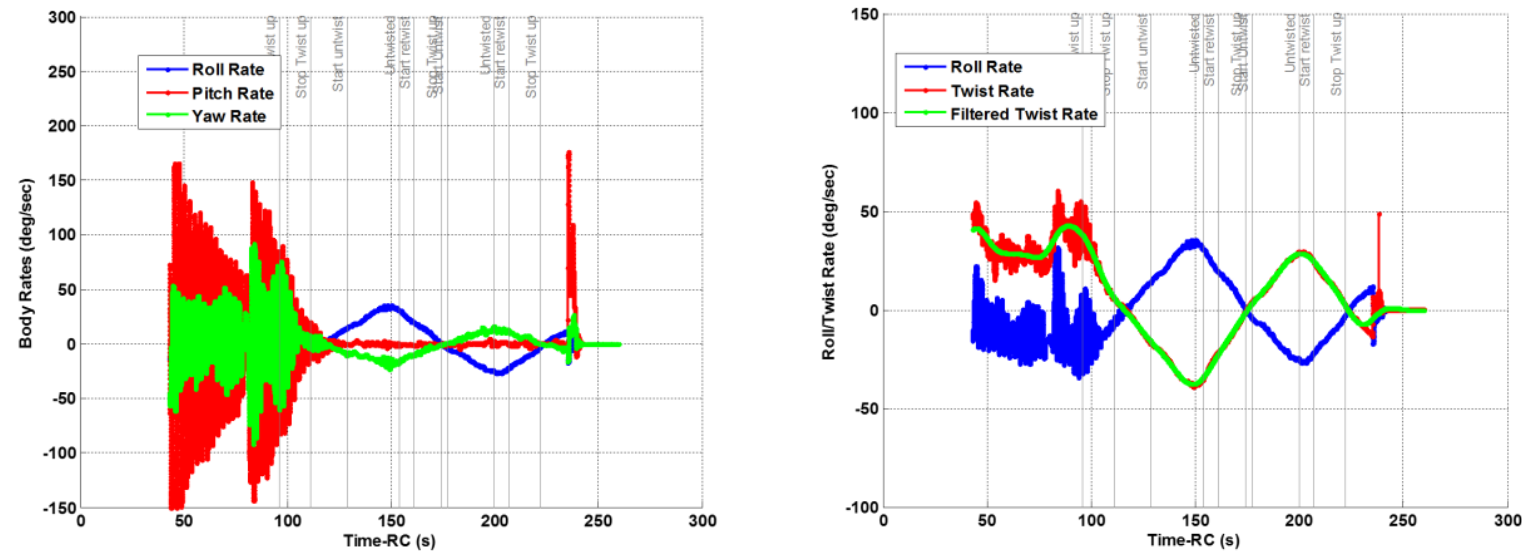

Figure 16. CDT-3-3 Rates.

The simulation was run and twist torque was again calculated for CDT-3-3 and CDT-3-5 as shown in the following figures.
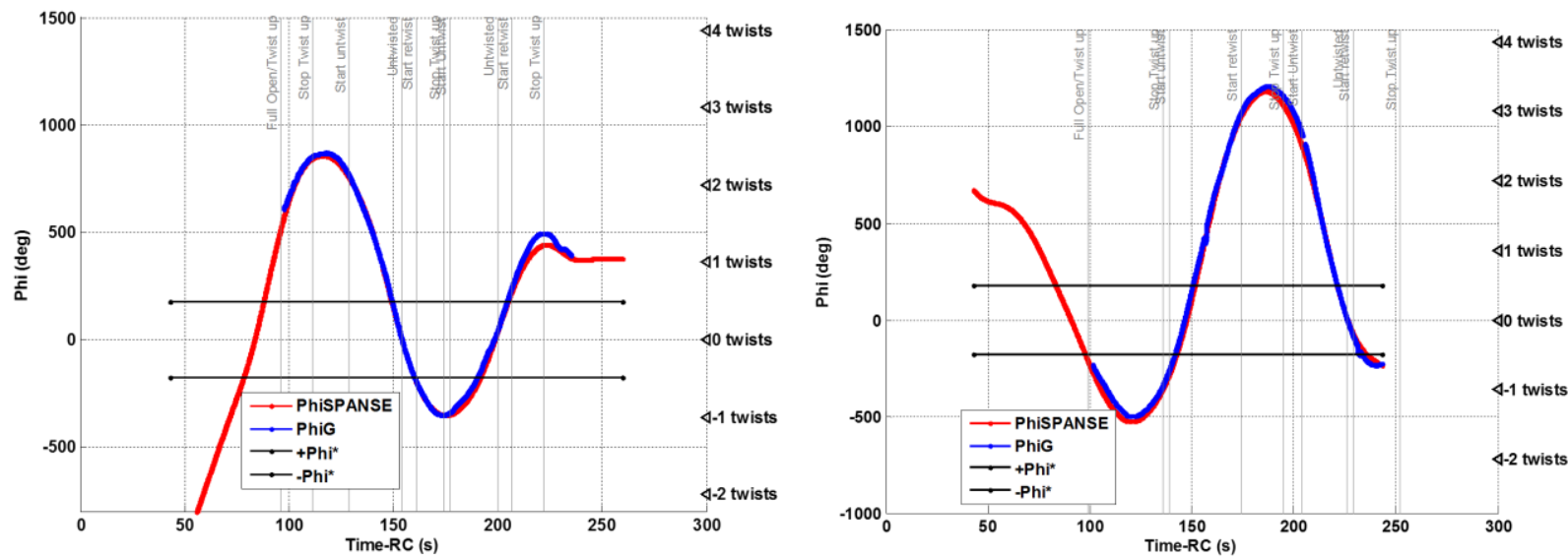

Figure 17. CDT-3-3 and CDT-3-5 (respectively) Twist Angles.
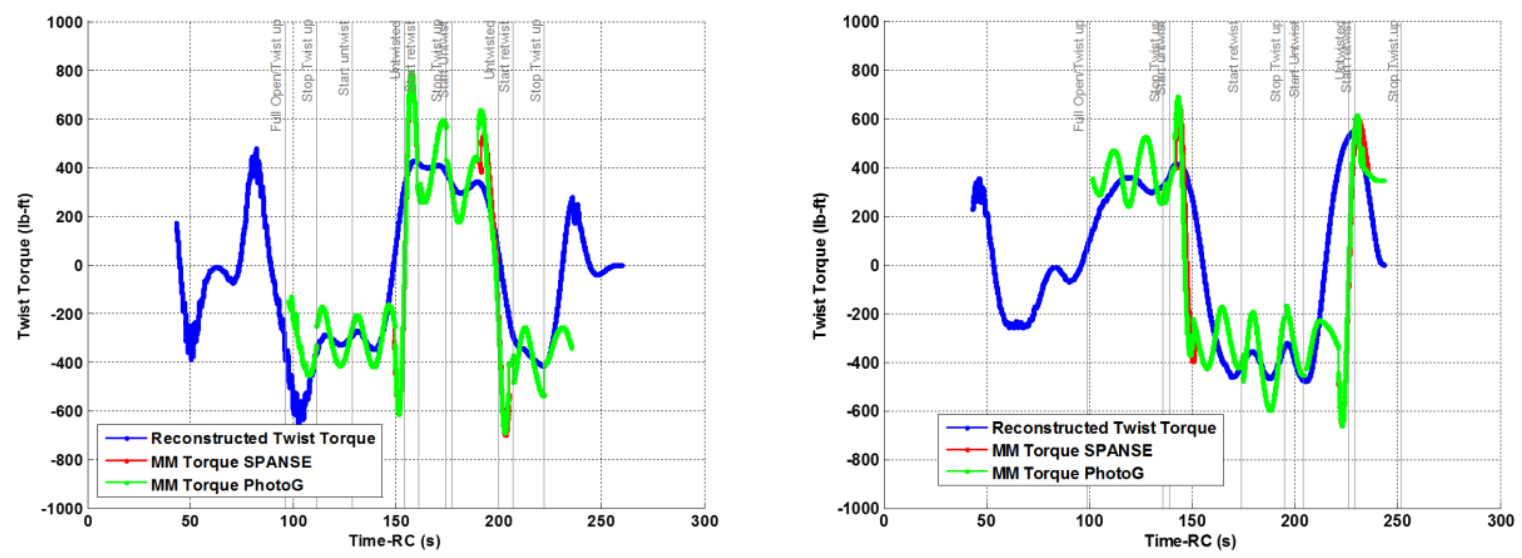

Figure 18. CDT-3-3 and CDT-3-5 (respectively) Twist Torques. 
As Fig. 18 shows the Model Memo Torque Equation (green and red traces) was a good match for the true torque (blue trace) and was conservative.

\section{Conclusion}

A successful reconstruction of twist torque in Main parachute risers is presented. The Model Memo Conservative Torque Equation for steel risers using photogrammetrics matches test data well. As shown in the twist angle in Fig. 14 photogrammetrics is more reliable due to accounting for relative position of the parachutes to the body. The Model Memo is conservative with respect to the test data, but is acceptable. The Model Memo $\mathrm{k}$ and $\mathrm{R}_{\mathrm{W}}$ parameters are correct for tests using steel risers, but will need to be re-evaluated for the new textile risers. If a closer match to test data is desired the $\mathrm{k}$ parameter would need to be varying based on time and twist position. It is concluded that the Model Memo equations and the simulation can accurately predict Main parachute riser twist torque and should be used in future tests.

\section{Acknowledgments}

The author wishes to acknowledge the contributions of Dr. Vladimir Drozd of Airborne Systems North America in deriving the Model Memo equations, Chris Madsen of NASA-JSC in the reconstruction of twist torque of CDT-31 and CDT-3-2, and Eric Ray (MRI Technologies) and John Davidson (GeoControl Systems, Inc.) of the JSC

Engineering Technology and Science contract (JETS) in the assistance and guidance in the development of the simulation.

\section{References}

${ }^{1}$ Ray, E. S., et al., Capsule Parachute Assembly System (CPAS) Engineering Development Unit Operating Modeling Parameters Version 13, JSC 65914 Rev I, ESCG-8400-CPAS-10-CPAS-MEMO-0069 Rev I, April 2014, JSC Engineering Technology and Science, Jacobs Engineering. 